\title{
Neuromodulación quirúrgica. Nuevos horizontes en Neurocirugía
}

\author{
F.J. Robaina Padrón
}

Unidad del Dolor Crónico y Neurocirugía Funcional. Hospital Universitario de Gran Canaria Doctor Negrín.Las Palmas de Gran Canaria.

Resumen

Objetivos. La neuromodulación quirúrgica se refiere al estudio de todas aquellas técnicas quirúrgicas que utilizan equipos implantables que liberan electricidad o sustancias químicas con la finalidad de modificar la transmisión de las señales nerviosas para conseguir inhibir, excitar o modular la actividad de grupos y redes neuronales y obtener un efecto terapéutico. La neuromodulación engloba diferentes aspectos científicos y tecnológicos por lo que puede definirse desde distintas ópticas.

Material y método. Desde el punto de vista de los aspectos quirúrgicos, la neuromodulación quirúrgica se define como: aquellas técnicas intervencionistas que alteran la transmisión de las señales neuronales mediante equipos implantables tanto eléctricos como químicos, con la finalidad de excitar, inhibir o modular la actividad de neuronas 0 redes neuronales para conseguir efectos terapéuticos. Una definición clínica es la que hace referencia a la utilización de forma reversible de la estimulación eléctrica o química aplicada al sistema nervioso para manipular su actividad con la finalidad de tratar algunos tipos específicos de dolor crónico, espasticidad, epilepsia, isquemia cardiaca, alteraciones de la motilidad del intestino y de la vejiga, lesiones del sistema nervioso y alteraciones del movimiento, visuales, auditivas o psiquiátricas Los neurocirujanos estamos actualmente perfectamente entrenados para la realización de un gran número de técnicas quirúrgicas de neuromodulación, justo cuando asistimos a un incremento muy significativo de las actividades biomédicas y de alta tecnología aplicadas al sistema nervioso central y periférico.

Conclusiones. La neuromodulación quirúrgica implica para los neurocirujanos entrar en una nueva neurocirugía, más allá de las clásicas técnicas de resección quirúrgica y de los procedimientos neuroablativos, para entrar en el campo de la neuroingeniería y el

Recibido: 30-08-07. Aceptado: 20-09-07 restablecimiento de las funciones neurológicas perdidas. Las posibles interrelaciones entre el cerebro y un computador (conexión cerebro-máquina) ya es un echo y ha sido utilizado en el campo de las neuroprótesis y la estimulación cerebral profunda. A la neurocirugía en general y a la española en concreto, se le presenta una oportunidad nueva de embarcarse en una travesía altamente tecnificada que nos llevará en pocos años a descubrir y aplicar nuevas formas de resolver los problemas neurológicos de muchos de nuestros pacientes gracias al empleo de las nuevas tecnologías quirúrgicas no lesivas.

PALABRAS CLAVE: Neuromodulación quirúrgica. Estimulación cerebral. Estimulación medular. Estimulación nervios periféricos. Interfase cerebro-máquina.

Surgical neuromodulation: new frontiers in neurosurgery

Summary

Objectives. Surgical neuromodulation refers to all those techniques that use implantable devices that discharge electricity or chemical substances that modify nerve signal transmission in order to achieve inhibition, excitation or modulation of the activity of neuronal groups and networks, and to achieve a therapeutic effect. Neuromodulation encompasses different scientific aspects and technologies which need to be defined.

Abreviaturas. CH: cluster Headache. DES: deep brain stimulation. FDA: food and drug administration. GABA: ácido gamma amino butírico. Gpi: globus pallidus internus. IFEES: Internacional Functional Eectrical Stimulation Society. INS: International Neuromodulation Society. NMQ: neuromodulación quirúrgica. PET: positron emission tomography. RNMf: resonancia nuclear magnética funcional. SENEMO: Sociedad Española de Neuromodulación. STN: núcleo subtalámico. TAC: tomografia axial computarizada. Vim: ventralis intermedius. WFNS: World Federation Neurosurgical Societies 
Material and method. From the surgical point of view, neuromodulation is defmed as: those intervention techniques that alter the transmission of neuronal signals using implantable electrical or chemical devices with the objective of stimulating, inhibiting or modulating the activity of neurones or neuronal networks to achieve therapeutic effects. A clinical definition makes reference to the use of reversible electrical or chemical stimulation of the nervous system to manipulate its activity in order to treat some specific types of chronic pain and conditions such as spasticity, epilepsy, cardiac ischemia, alterations in the motility of the intestine and of the bladder, lesions of the nervous system, and alterations in mobility, visual, auditory or psychiatric status. Neurosurgeons have been well trained to perform a great number of surgical techniques of neuromodulation, even including helping to significantly increase biomedical activities and the application of high technology to the central and peripheral nervous system.

Conclusions. Surgical neuromodulation encourages the neurosurgeon to go also away from the classical techniques of surgical resection and neuroablative procedures, and to enter into the new field of neuroengineering to re-establish lost neurological functions. The inter-relationship between the brain and the computer (brain-machine interface) has already occurred and has been applied in the field of neuroprosthetics and deep brain stimulation. For neurosurgery in general and for Spain in particular, this represents a new opportunity to embark on a high technology path that would involve years of research but, applying these new, non-invasive surgical techniques would help resolve the neurological problems of many of our patients.

KEY WORDS: Surgical neuromodulation. Deep brain stimulation. Spinal cord stimulation. Peripheral nerve stimulation. Brain-computer interface.

\section{Introducción}

La neuromodulación quirúrgica (NMQ) se refiere inicialmente a todas aquellas técnicas quirúrgicas que utilizan equipos implantables que liberan electricidad o sustancias químicas con la finalidad de alterar la transmisión de las señales nerviosas para conseguir inhibir, excitar o modular la actividad de grupos y redes neuronales y obtener un efecto terapéutico. Los neurocirujanos estamos perfectamente entrenados para la realización de un gran número de técnicas quirúrgicas de neuromodulación ahora que asistimos a un incremento muy significativo de las actividades biomédicas y de alta tecnología aplicadas al sistema nervioso. La neuromodulación implica para los neurocirujanos entrar en una nueva neurocirugía, más allá de las clásicas técnicas de resección quirúrgica y de los procedimientos neuroablativos, para entrar en el campo de la neuro-ingeniería y el restablecimiento de las funciones neurológicas perdidas ${ }^{100}$. La NMQ se desarrolla en un campo de actividades multidisciplinares donde confluyen las neurociencias, las técnicas biomédicas y biotecnológicas, no sólo para ayudar a los pacientes que es su principal objetivo, sino también para profundizar en el conocimiento de cómo el sistema nervioso modula y controla al resto del organismo.

La Sociedad Internacional de Neuromodulación (International Neuromodulation Society-INS) ha nacido para aglutinar todos los esfuerzos para favorecer la investigación, la diseminación de los conocimientos y estimular la educación y la aplicación de las técnicas de neuromodulación. La INS está directamente asociada a la Sociedad Internacional de Estimulación Eléctrica Funcional (Functional Electrical Stimulation Society-IFEES), que se orienta hacia la investigación y la aplicación de la estimulación eléctrica en el campo de la medicina rehabilitadora ${ }^{59}$.

La Federación Mundial de Sociedades Neuroquirúrgicas (World Federation Neurosurgical Societies-WFNS) ha visto el potencial de este nuevo campo y ha creado el comité de neuromodulación ${ }^{99}$.

Como hemos visto, la neuromodulación engloba diferentes aspectos científicos y tecnológicos, por lo que puede definirse desde distintas ópticas. Así, comenzando por los aspectos biológicos, la neuromodulación, se define como aquel proceso químico, neuronal o de redes neuronales, que excita, inhibe o modula neuronas, redes neuronales adyacentes o remotas, para que estas últimas, emitan respuestas que se adapten de una mejor manera a las demandas ambientales de un determinado organismo para asegurar su supervivencia. Desde los aspectos biotecnológicos, se define la neuromodulación, como aquel campo de la ciencia, la medicina y la bioingeniería, que estudia las tecnologías implantables y no implantables, eléctricas o químicas, que se aplican con la intención de mejorar la calidad de vida de las personas que sufren trastornos neurológicos. Desde el punto de vista de los aspectos clínicos, se han acuñado diferentes definiciones tales como: a) la ciencia que estudia cómo procedimientos eléctricos, químicos y mecánicos, pueden modular o cambiar el funcionamiento del sistema nervioso central y periférico; b) una forma de terapia en la que se activan señales neurofisiológicas con la finalidad de conseguir un efecto terapéutico mediante la modificación de la actividad del sistema nervioso; c) la modificación con fines terapéuticos de la actividad del sistema nervioso central, periférico o autonómico, mediante la aplicación de estímulos eléctricos o farmacológicos, empleando sistemas implantables o no; d) la utilización de forma reversible de la estimulación eléctrica o química aplicada al sistema nervioso para manipular su actividad con la finalidad de tratar 
algunos tipos específicos de dolor crónico, espasticidad, epilepsia, isquemia cardiaca, alteraciones de la motilidad del intestino y de la vejiga, lesiones del sistema nervioso y alteraciones del movimiento, visuales, auditivas o psiquiátricas $^{78}$.

Desde el punto de vista de los aspectos quirúrgicos, la $\mathrm{NMQ}$, se define como: aquellas técnicas intervencionistas que alteran la transmisión de las señales neuronales mediante equipos implantables tanto eléctricos como químicos, con la finalidad de excitar, inhibir o modular la actividad de neuronas o redes neuronales para conseguir efectos terapéuticos. Generalmente, los especialistas clínicos relacionados con las técnicas de neuromodulación han sido los anestesiólogos, los neurocirujanos, neurofisiólogos, neurólogos, cardiólogos, y traumatólogos, pero debido a los efectos sistémicos de estas técnicas, otras especialidades médicas se incorporaran a este nuevo campo multidisciplinar de la medicina. La neuromodulación, aunque afecta como vemos a muchas especialidades médicas, biomédicas y biotecnológicas, está considerada actualmente como el campo de mayor crecimiento de la medicina actual $1^{59,60,100}$.

La reciente publicación del primer libro de texto sobre neuromodulación quirúrgica (Operative Neromodulation $)^{80,81}$, nos permite, basándonos en su extenso contenido neuroquirúrgico, reclamar para la neurocirugía una presencia fundamental tanto en la realización de las investigaciones como en la práctica de las técnicas que como puede apreciarse están desarrollándose a gran velocidad en estos momentos en la comunidad científica y tecnológica ${ }^{80,81}$.

Como evidencia Sakas y cols. ${ }^{100}$, ha sido el manejo del dolor crónico el gran éxito de la neuromodulación hasta la fecha, concretamente la estimulación eléctrica de los cordones posteriores, existiendo una gran cantidad de publicaciones que demuestran su eficacia. España, no se ha quedado a la saga, sino que ha participado con numerosos grupos de investigadores tanto desde el campo de la anestesiología como de la neurocirugía los cuales más adelante iremos mencionando en los diferentes apartados de esta revisión. Igualmente, los españoles, hemos participado y lo seguimos haciendo en los avances conseguidos con la infusión espinal de medicamentos tanto para el manejo del dolor crónico como de la espasticidad, autores que igualmente mencionaremos también más adelante en el apartado correspondiente.

La finalidad del presente artículo de revisión radica en la necesidad a mi juicio de exponer ante los neurocirujanos españoles jóvenes y no tan jóvenes, las nuevas técnicas de NMQ en las que muy posiblemente algunos ya están participando o se verán avocados a participar a corto plazo, ya que la presión de otras especialidades como la neurología, la neurofisiología, la rehabilitación y la psiquiatría, entre otras, demandarán la realización de dichas técnicas en alguno de sus pacientes. No se trata en este artículo de revisión de describir en profundidad las diferentes técnicas de NMQ, sino presentar la situación actual de muchas de ellas clásicas y algunas técnicas emergentes novedosas para tener una idea de la cantidad de intervenciones funcionales que se avecinan y que se realizan o se realizarán en los servicios de neurocirugía con la colaboración de otras especialidades ya descritas, o colaborando con otras nuevas especialidades como la urología o la cirugía digestiva como ya veremos más adelante.

Posiblemente, debido al enorme auge que se espera de muchas de estas técnicas, los pacientes candidatos a las mismas deberían ser remitidos a hospitales con redes de neurociencias bien desarrollas, con equipamiento tecnológico adecuado y personal con especial dedicación o interés en la NMQ. Por lo tanto, aquellos servicios de neurocirugía que no estén preparados pero si interesados, ya saben que deben irse equipando técnicamente y dotándose de personal con especial dedicación a este campo emergente de nuestra especialidad.

Un aspecto importante que da una idea del auge de las técnicas de NMQ estriba en las previsiones económicas referentes al número de equipos implantables que se utilizarán en los próximos años. Se estima, que a finales del año 2008, el mercado de la neuromodulación ascienda en el mundo hasta "dos billones de dólares" $"$.

En España, se ha constituido recientemente el capítulo español de la Internacional Neuromodulation Society (INS), denominado Sociedad Española de Neuromodulación (SENEMO), que nace con la intención de aglutinar y colaborar con todas las especialidades médicas que realizan o realizarán procedimientos de neuromodulación, así como con las empresas de bioingeniería que promueven investigación y desarrollo en este campo ${ }^{59}$. Deseamos que al igual que la WFNS ha creado puentes con la INS a nivel internacional, aquí en España, esperamos que ocurra lo mismo entre sociedades que abarcan muchos campos de aplicación e investigación en neuromodulación.

$\mathrm{Al}$ adentrarnos en la descripción de las técnicas de NMQ, comenzaremos analizando las nuevas técnicas aplicadas en el sistema nervioso central tanto a nivel cerebral como medular. Posteriormente, veremos cuáles son las nuevas aplicaciones de las mismas en el sistema nervioso periférico tanto en el campo del dolor como en la novedosa esfera urológica y en la proctología. El campo de las neuroprótesis y la rehabilitación funcional del lesionado medular y cerebral también será motivo de una breve puesta al día, tanto de su situación actual como de las expectativas a corto plazo.

Destacar ya desde este momento que el mayor número de innovaciones técnicas se está produciendo en el área cerebral, lo que hará que los neurocirujanos seamos mucho más necesarios que lo hemos sido o somos actualmente 


\section{Tabla I}

Aplicaciones rutinarias de la Estimulación Cerebral Profunda

\section{Enfermedad de Parkinson}

- Núcleo ventralis intermedius (Vim), globus pallidus internus (GPi), núcleo subtalámico $(\mathrm{STN})$, zona incerta

\section{Distonía}

- Globus pallidus internus (GPi)

Temblor (esencial, cerebeloso, esclerosis múltiple)

- Nucleus ventralis intermedius (Vim), zona incerta

Coreoatetosis

- Nucleus ventralis intermedius (Vim), nucleus ventro-oralis posterior (Vop), globus pallidus internus (Gpi), zona incerta

Dolor Neuropático/Dolor por Deaferentización

- Núcleo ventro-postero-medial (VPM), núcleo ventro-postero-lateral (VPL), Núcleo centro mediano (CM), sustancias gris periacueductal (PAG), sustancia gris Periventricular (PVG)

Cita bibliográfica ( 34)

Tabla II

Otras aplicaciones experimentales de la Estimulación Cerebral Profunda

Epilepsia

-Tálamo anterior, núcleo centro mediano (CM), núcleo subtalámico (STN), hipocampo

Cluster Headache

- Hipotálamo posteromedial

Trastorno obsesivo compulsivo

- Extremidad anterior de la cápsula interna, núcleo accumbens

Síndrome de Tourette

- Núcleo centro mediano (CM), núcleo ventro-oral-interno (Vol), globus pallidus intemus (GPi), sustancia gris periventricular (PVG)

Depresión

- Extremidad anterior de la cápsula interna, núcleo accumbens, cíngulo anterior

Adicción a drogas

- Núcleo accumbens

Conducta agresiva

- Hipotálamo posteromedial

Obesidad

- Hipotálamo anterior

Cita bibliográfica ( 34$)$ 
para la realización de las intervenciones quirúrgicas de NMQ.

\section{Neuromodulación cerebral}

Las técnicas de neuromodulación cerebral han experimentado un gran avance en los últimos años gracias a la llegada de los sistemas de cirugía guiada por la imagen (neuronavegación) ${ }^{65}$. Prácticamente todos Ios clásicos targets para termolesiones con radiofrecuencia son ahora estimulados eléctricamente. Las nuevas técnicas de neuromodulación se caracterizan por su reversibilidad, bilateralidad, no son lesivas, presentando una baja incidencia de complicaciones y menor riesgo que las técnicas ablativas $^{34}$. Las técnicas estereotácticas clásicas con marco fijo están siendo reemplazadas por la cirugía sin marco (Frameless), con excelentes resultados en términos de precisión, fiabilidad, complicaciones y tiempo empleado en la realización de los implantes de los electrodos ${ }^{49}$.

La Food and Drug Administration (FDA) norteamericana, ha aprobado el empleo de la estimulación cerebral profunda (DBS) uni o bilateral del núcleo ventralis intermedius (Vim) del tálamo en caso de temblor esencial y temblor del Parkinson, así como la estimulación uni o bilateral del globus pallidus internus (GPi) y el núcleo subtalámico (STN) en la enfermad de Parkinson ${ }^{11,12,73}$. Estas técnicas no están exentas de complicaciones incluyendo algunas serias como las hemorrágicas debido generalmente al empleo de los microrregistros y a la curva de aprendizaje necesaria $^{14,40,47,48,79}$. La tabla I, muestras las indicaciones actuales de la DBS donde existe un consenso general en su aplicación y selección de pacientes y, la tabla II, muestra las indicaciones en que experimentalmente está siendo utilizada la DBS sin que exista definitivamente un consenso. No obstante, ambas tablas dan una idea clara del amplísimo panorama de aplicaciones de la DBS que se abre ante los neurocirujanos.

Una nueva aplicación en el manejo del dolor crónico mediante DBS es su empleo para el control del dolor del Cluster Headache $(\mathrm{CH})$ en su fase crónica, rebelde a todo tipo de tratamiento médico, y que puede persistir meses y años. En un grupo de pacientes con este diagnóstico se han implantando electrodos a nivel del hipotálamo ipsilateral, ya que estudios mediante resonancia nuclear magnética funcional y/o tomografía por emisión de positrones (PET) durante las crisis de dolor demuestran la activación de esta zona $^{63,103,107 .}$.

Las indicaciones de la DBS en otros trastornos del movimiento incluyen el temblor, las diskinesias, bradikinesias, rigidez, distonía y alteraciones de la marcha ${ }^{26.98}$. La DBS se emplea actualmente en algunos casos de dolor neuropático ${ }^{50}$, cluster headache $e^{8.36,63,107}$, epilepsia ${ }^{12,25,115}$, trastorno obsesivo compulsivo ${ }^{4,6}$, sindrome de Tourette ${ }^{120}$, depresión $^{52,69}$, conducta violenta ${ }^{37}$, otras alteraciones psiquiátricas ${ }^{96} \mathrm{y}$ en casos de coma por estado vegetativo ${ }^{110}$.

La estimulación de la corteza motora está siendo utilizada para el tratamiento de algunos casos de dolor neuropático de predominio en cara y mano con muy buenos resultados y constituye una técnica que se realizará cada vez con mayor frecuencia a medida que se difunda su conocimiento entre los futuros médicos referidores ${ }^{20,39,54,76,85,86,111}$. La estimulación de la corteza motora se está explorando también como target para el control de los síntomas de la enfermedad de Parkinson ${ }^{22}$.

La corteza auditiva ha sido estimulada eléctricamente para el tratamiento del tinnitus incontrolable con muy buenos resultados clínicos y escasas complicaciones menores $^{27}$.

Otro aspecto novedoso de la neuromodulación cerebral lo encontramos en el manejo de la epilepsia. La estimulación del nervio vago cada vez va cobrando mayor presencia en el manejo de las crisis complejas no susceptibles de resección quirúrgica ${ }^{5}$. Igualmente, la estimulación eléctrica del nervio vago, se ha indicado y se aplica actualmente en algunos casos de depresión severa que no responde al tratamiento farmacológico ${ }^{83}$. Ambas indicaciones de la estimulación del nervio vago han sido aprobadas por la FDA norteamericana.

La aplicación de la estimulación cerebral profunda (DBS) en el campo de la epilepsia también está aumentando tal y como lo demuestran los artículos que actualmente se publican no sólo en el campo de los estudios de los mecanismos neurofisiológicos, sino también en el de sus verdaderas aplicaciones clínicas, preconizándose incluso la implantación de electrodos en distintas localizaciones cerebrales para inhibir las crisis mediante la estimulación crónica de la zona ${ }^{55}$.

Actualmente está comercializado un dispositivo electrónico subcutáneo alojado en una pequeña craniectomía, el cual mediante dos electrodos implantados uni o bilateralmente en diferentes áreas del cerebro en pacientes no candidatos a resección quirúrgica, es capaz de detectar con un electrodo el inicio de la crisis y automáticamente suprimirla estimulando la zona con un segundo electrodo ${ }^{35}$.

Mención especial se merece por novedosa y trascendental la aplicación de la $D B S$ en psiquiatría. La psicocirugía ha experimentado un cierto auge en los últimos 10 años coincidiendo con la relativa facilidad para realizar la DBS gracias a los sistemas de cirugía por imagen, la facilidad para emplear los microrregistros y la fiabilidad de los productos que se implantan. Actualmente la DBS en psiquiatría debe entenderse como una modalidad de tratamiento y no como una alternativa terapéutica en sentido estricto ya que se puede conseguir una mejoría en los síntomas psiquiátricos en un limitado número de pacientes sin producir 
una lesión permanente ${ }^{101}$. Se han propuesto diferentes targets, algunos experimentales como el núcleo accumbens y otros con aplicaciones clínicas directas como es la DBS en el pedúnculo talámico inferior en caso de depresión severa y trastorno obsesivo compulsivo ${ }^{52,114}$.

El hipotálamo medial ha sido usado clínicamente para tratar episodios severos de Cluster Headache al igual que síndromes de alteración de la conducta, generalmente agresivos y de etiología connatal ${ }^{38}$. Ya hemos mencionado anteriormente la utilidad de la estimulación eléctrica del nervio vago en el cuello para el control de la depresión al igual que algunos tipos de epilepsia. En julio de 2005 la FDA norteamericana aprobó el empleo de un estimulador vagal para el tratamiento a largo plazo de la depresión crónica recurrente en pacientes mayores de 18 años que no han respondido al empleo de cuatro antidepresivos diferentes ${ }^{83}$.

Destacar, finalmente, en este apartado, que los españoles también hemos aportado nuestro conocimiento para el desarrollo y divulgación de las técnicas y aplicaciones de la DBS a lo largo de los últimos 10 años, tal y como se demuestra en la bibliografía que puede consultarse al respecto $^{2,3,9,10,30-33,41,44-46,71,102}$.

\section{Neuromodulación medular}

Son ya clásicas las aplicaciones de la neuromodulación eléctrica y química de la médula espinal para el control del dolor crónico de diferentes etiologías y la espasticidad de origen traumático o degenerativa. Los españoles hemos participado desde los inicios de estas técnicas quirúrgicas en el desarrollo y aplicación de las mismas, tanto en las técnicas de estimulación eléctrica medular como en la de infusión espinal de medicamentos ${ }^{1,7,15-17,23,42,88-90,116}$.

Recientemente, se han publicado nuevos estudios que corroboran todos los resultados publicados a lo largo de casi 30 años en el campo del manejo del dolor crónico y, donde como ya mencioné anteriormente, los españoles, hemos participado activamente tanto clínica como experimentalmente ${ }^{24,61,95,112}$.

Otro de los aspectos novedosos de la estimulación eléctrica medular es su aplicación en el campo del dolor visceral abdominal, en situaciones posteriores a pancreatitis, neuromas en pared abdominal, esplenectomías postraumáticas y dolor abdominal difuso postquirúrgico. La estimulación de los cordones posteriores de la médula espinal entre D5 y D8 (niveles vertebrales), permite controlar este tipo de dolor altamente incapacitante ${ }^{58}$.

Existen además aplicaciones emergentes que muestran la utilidad de la estimulación eléctrica de los cordones posteriores de la médula espinal a nivel cervical con la finalidad de mejorar la oxigenación cerebral y el metabolismo locorregional, abriendo expectativas en el manejo de la isquemia cerebral e incluso la aplicación de esta técnica en el tratamiento radio y quimioterápico de los tumores cerebrales malignos ${ }^{91}$. Este último aspecto está pendiente de un estudio multicéntrico que pueda confirmar los hallazgos y resultados obtenidos por los autores de los únicos trabajos publicados hasta la fecha con esa indicación ${ }^{23,92}$.

La estimulación eléctrica medular epidural a nivel cervical se ha aplicado también en pacientes en estado vegetativo persistente postraumático. Los estudios recientemente publicados en pacientes jóvenes con atrofia cerebral y con flujo sanguíneo cerebral disminuido valorado mediante PET (superior a 20m1/100gr), hablan de un $80 \%$ de "resultados positivos" en función del aumento del flujo cerebral y del grado de alerta que presentaba cada paciente antes de ser estimulado ${ }^{74}$.

\section{Neuromodulación nervios periféricos}

Este es un campo en franco crecimiento para la búsqueda de soluciones a casos complejos de dolor crónico. El empleo de la estimulación de nervios periféricos directamente en el tejido subcutáneo utilizando electrodos implantados percutáneamente, ha permitido aplicar esta técnica en casos rebeldes de neuritis postherpética en la primera y segunda ramas del trigémino con resultados bastante esperanzadores. Las ventajas iniciales de estas técnicas son su reversibilidad y posibilidad de bilateralidad. Se puede conseguir alivio del $50 \%$ de la intensidad del dolor en el $70 \%$ de los pacientes ${ }^{53}$. Otras aplicaciones de la estimulación de los nervios periféricos en el espacio subcutáneo a nivel facial, lo constituyen otros casos de dolor de origen neuropático postraumático o postquirúrgico en zonas inervadas por la primera y segunda ramas del trigémino $^{105,106}$.

Uno de los aspectos novedosos de la aplicación de estas técnicas de estimulación subcutánea muy útiles en la práctica diaria es su aplicación en el complejísimo manejo del tratamiento médico y quirúrgico del Cluster Headcache (CH). Un 10\% de los pacientes afectos de $\mathrm{CH}$ desarrollan una variante crónica, que puede extenderse más allá de un año sin remisiones y no responden al tratamiento médico con triptanes inyectables o nasales, inhalaciones de oxígeno, esteroides, verapamil, metisergida, o carbonato de litio. Los bloqueos anestésicos o la radiofrecuencia del ganglio esfenopalatino en la fosa pterigomaxilar no consiguen aliviar el dolor en muchos de estos casos de $\mathrm{CH}$ en su variante crónica (experiencia personal del autor).

Como ya vimos en el apartado dedicado a la DBS, esta modalidad terapéutica puede emplearse también en estos casos aunque implica asumir mayores riesgos de generar lesiones neurológicas permanentes e incluso riesgo de muerte por hemorragia intracerebral ${ }^{103}$. Ello ha motivado el buscar nuevas alternativas terapéuticas dentro del campo de la NMQ. Así, parece que se están obteniendo buenos 
resultados en el $\mathrm{CH}$ rebelde mediante la estimulación eléctrica subcutánea de los nervios suboccipitales. Los estudios clínicos iniciados por Weiner en 1992 demostraron que el efecto analgésico a largo plazo persistía en muchos casos de cefalea cervicogénica o cefalea occipital ${ }^{122}$, dando paso a la aplicación de la técnica en el $\mathrm{CH}$ rebelde. Artículos de reciente publicación demuestran cómo se consiguen elevados porcentajes de alivio persistente del dolor a largo plazo en este tipo de pacientes ${ }^{18,66}$. El mecanismo de acción de la neuromodulación suboccipital propuesto consiste en la activación de la región dorsal superior del tronco cerebral registrada mediante PET en pacientes sometidos a este tipo de terapia ${ }^{68}$. Es posible, además, que la estimulación periférica de los nervios suboccipitales induzca cambios en el flujo sanguíneo de las regiones activadas y a su vez influya en los mecanismos trigeminovasculares a nivel de la médula cervical alta ${ }^{122}$.

Otro aspecto de la NMQ a nivel del sistema nervioso periférico en la que los neurocirujanos podemos participar multidisciplinariamente es colaborando con los urólogos y los proctólogos en sus respectivos campos (bastante alejados por cierto de nuestro campo principal que es el cerebro y la médula espinal), pero ayudando a resolver casos muy complejos de dolor crónico e incontinencia urinaria o rectal entre otros. La estimulación eléctrica de las raíces sacras por vía transforaminal permite afrontar los trastornos neurógenos de la vejiga tanto la hipertonía como la incontinencia urinaria ${ }^{18}$. La incontinencia fecal es otra de las aplicaciones emergentes de este tipo de neuromodulación periférica ${ }^{87}$. También se aplica la estimulación de las raíces sacras para aumentar la función sexual, aunque la estimulación en estos casos no es transforaminal sacra sino epidural lumbar con electrodos localizados a nivel L1-L2 ${ }^{70}$. En el campo de la cirugía invasiva no percutánea como la que hemos estado describiéndo a nivel urológico, destacan las rizotomías posteriores combinadas con la estimulación eléctrica de las raíces anteriores sacras, mediante laminectomías L3-S1 e implante de electrodos directamente sobre las raíces anteriores S2-S4. Los resultados descritos en estudios de neurorehabilitación urológica son altamente favorables y nuestra colaboración como neurocirujanos para la realización de este tipo de cirugía es lógicamente indispensable $e^{62.117}$.

\section{Neuroprótesis}

Desde hace más de 20 años se están desarrollando aplicaciones de la microelectrónica a los implantes nerviosos desarrollando neuroprótesis motoras y sensitivas. Se han estado describiendo aplicaciones de microsistemas electrónicos en los nervios periféricos y en el sistema nervioso central, como es el caso de las prótesis visuales y la intrigante interfase cerebro-máquina que más tarde desarrollaremos en otro apartado ${ }^{108}$. El restablecimiento de las funciones motoras y sensitivas perdidas tras una lesión medular o cerebral es un campo que está siendo seriamente explorado y donde los neurocirujanos neuromoduladores tendrán que colaborar activamente. En el campo de las neuroprótesis motoras destacan los marcapasos frénicos que permiten respirar a pacientes con lesiones medulares altas. Igualmente los implantes en las raíces anteriores en los lesionados medulares para favorecer la micción ya des$\operatorname{critas}^{62,117}$, o el restablecimiento de la motilidad del brazo y la mano en algunos lesionados medulares por encima de C4 mediante sistemas que canalizan los impulsos nerviosos. Recientemente se han publicado resultados favorables para realizar la pinza de la mano en tetrapléjicos ${ }^{75,84,97}$.

Como ya hemos comentado previamente en este apartado, la restitución de las funciones sensoriales es otro de los caballos de batalla de las técnicas de NMQ. Ejemplo de ello lo vemos reflejado en el campo de la audición y en el de la visión. En el primero, podemos distinguir claramente dos tipos diferentes de prótesis: los implantes cocleares y los del tronco cerebral, estando indicados cada uno de ellos en pacientes diferentes. El electrodo de tronco cerebral es una modificación del implante coclear y la necesidad de este tipo de implante queda para aquellos pacientes completamente sordos debido a una lesión completa del VIII par durante una cirugía para extirpar neurofibromas bilateralmente, o en pacientes con neurofibromatosis tipo $2^{29}$. Nuestro país ha participado con algunos autores en la experiencia adquirida en el mundo en el implante de tronco cerebral y actualmente se realizan estudios experimentales con simios en la Universidad de Navarra ${ }^{21}$.

No podemos dejar de comentar nuevamente en este campo de la neuromodulación de la audición en general, los buenos resultados que comienzan a publicarse en relación a la estimulación de la corteza auditiva en la región parietal que se observa cómo se activa en la resonancia nuclear magnética funcional (RNMf) durante las crisis de tinnitus incontrolable ${ }^{27}$.

El campo de las neuroprótesis visuales está siendo claramente explorado tal y como se puede observar en publicaciones recientes en el campo de la neuro-oftalmología. La degeneración macular y la retinitis pigmentosa son las dos causas más frecuentes de pérdida de visión. El ojo biónico está muy cerca de ser implantado en humanos ${ }^{119}$.

Recientemente, nos consta (información recibida por el autor por e-mail), que la Cleveland Clinic, USA (Functional Electrical Stimulation Center, FES), está buscando investigadores para desarrollar un programa independiente de prótesis neurales en el territorio cabeza-cuello. Concretamente, quieren desarrollar el campo de las neuroprótesis para restablecer la voz, la parálisis facial y el mecanismo de la deglución en pacientes lesionados neurológicos.

Los aspectos bioéticos de todas estas nuevas tecnolo- 
gías de bioingeniería deberán ser resueltos adecuadamente, ya que además de todo lo descrito, se empieza a hablar de "aumentar las capacidades del sistema nervioso de personas sanas" 14 . Existen aspectos sociales y legales que deberán ser resueltos adecuadamente a medida que avancemos en la investigación y desarrollo de este fascinante campo de las neuroprótesis y la interfase cerebro- máquina ${ }^{109}$.

\section{Indicaciones emergentes}

Las diferentes líneas de investigación que se están siguiendo en la NMQ están aportando nuevas aplicaciones tanto a nivel del sistema nervioso como en el campo de la bioingeniería aplicada. A nivel medular, como ya hemos mencionado previamente en su apartado correspondiente, destacan como aplicaciones emergentes la utilización de la estimulación de los cordones posteriores a nivel cervical para incrementar el flujo y la oxigenación cerebral en humanos, tanto en el accidente cerebrovascular cómo en los tumores cerebrales malignos, con una finalidad terapéutica $^{91,92}$. Igualmente, mencionamos el empleo de la estimulación medular cervical alta en pacientes con niveles de conciencia alterada en estado casi vegetativo ${ }^{74}$.

A nivel cerebral, destacan los fascinantes estudios que hablan del empleo de la DBS para el control y estabilización de la tensión arterial (hipertensión arterial e hipotensión ortostática), sin la necesidad de recurrir a medicación que pudiera derivar en fallos cardiacos. La estimulación mediante electrodos implantados en la sustancia gris periacueductal pueden conseguir efectos beneficiosos en el campo cardiovascular en casos muy complejos $^{43}$. Igualmente, destaca la aplicación de los "estudios neurofisiológicos tridimensionales" durante los procedimientos de DBS en humanos, integrando toda la información derivada de la RNM, la TAC, los atlas cerebrales tridimensionales y los microrregistros, permitiendo optimizar de esta manera el implante definitivo del electrodo $^{19,72}$. La nanotecnología también ha llegado al campo de la DBS mediante la fabricación de electrodos de fibra de carbono, permitiendo en el futuro estimular una sola célula al mismo tiempo que podremos detectar las variaciones de los diferentes neurotransmisores como la dopamina, glutamato y el ácido gamma-aminobutírico (GABA) en el espacio extracelular ${ }^{64}$. Los modelos computarizados -neuromodulación computacional-, se están empleando para aumentar nuestro conocimiento sobre cómo la información es procesada en las diferentes estructuras cerebrales como por ejemplo los ganglios basales y sus ulteriores interacciones con el resto del cerebro ${ }^{77}$. Igualmente, las simulaciones de la distribución de los campos eléctricos durante la estimulación de la corteza motora permite conocer cómo se distribuyen y se modifican los mismos en función de las diferentes variables estudiadas tales como la polaridad de los electrodos o la presencia de mayor o menor cantidad de líquido cefaloraquídeo ${ }^{67}$. Se están desarrollando también modelos computarizados del envejecimiento cerebral, que permiten estudiar las diferentes situaciones que se pueden presentan en los fenómenos de envejecimiento cerebral ${ }^{104}$.

\section{Interfase cerebro-máquina}

He dejado para el final este llamativo apartado que ya hemos mencionado en varias ocasiones en líneas anteriores ya que abre unas perspectivas actualmente desconocidas. Las posibles interrelaciones entre el cerebro y un computador han sido ya exploradas y utilizadas en el campo de las neuroprótesis y la DBS como hemos analizado previamente en este artículo. Recientemente, hemos asistido a la publicación de algunos artículos que muestran los resultados obtenidos en pacientes con accidentes cerebrovasculares de tronco a los que se les ha implantado lo que se ha denominado " $3^{a}$ generación de implantes cerebrales", que les ha permitido mover un cursor en un pantalla de ordenador ordenándolo mentalmente. Los electrodos de esta nueva $3^{\mathrm{a}}$ generación se denominan "electrodos neurotróficos", ya que son de cristal y van rellenos de dos filamentos de oro y un componente neurotrófico. Se implantan en la corteza motora en el área de máxima actividad detectada por RNMf. El factor neurotrófico estimula el crecimiento del tejido neural en el interior del tubo-electrodo de cristal. Este ejemplo demuestra que es posible acoplar el cerebro y/o la médula espinal a un computador para conseguir una determinada acción. Hoy existen en el mercado diferentes tipos de electrodos para este propósito ${ }^{56,57}$. Cuando este tipo de electrodos se implanten en lesionados medulares, será posible conseguir una gran cantidad de efectos beneficiosos, algunos de ellos impensables hasta ahora tales como: reeducación cerebral; prevención de deformidades vertebrales; tratamiento del dolor; manejo de la vejiga; alivio de la espasticidad; prevención de úlceras cutáneas; restablecimiento de funciones sexuales; mejoría de la capacidad de movimiento y otras más ${ }^{121}$. En este extenso campo de las interacciones cerebro-máquina deberá caminarse con sumo cuidado y resolviendo las cuestiones éticas que vayan presentándose ante cada nuevo reto.

\section{Conclusiones}

No es mi intención extenderme mucho en las conclusiones ya que el que haya llegado hasta este punto habrá sacado las suyas propias al vislumbrar el alto potencial de las técnicas de neuromodulación quirúrgica para el desarrollo de nuestra especialidad, puesto que este artículo es exclusivamente de carácter informativo. No obstante, sí quisiera decir, que a la neurocirugía en general y a la espa- 
ñola en concreto, se le presenta una nueva oportunidad de embarcarse en una travesía altamente tecnificada que nos llevará en pocos años a descubrir nuevas formas de resolver los problemas neurológicos de muchos de nuestros pacientes, aplicando al cerebro y/o a la médula espinal (al sistema nervioso en general), nuevas tecnologías neuromoduladoras no lesivas.

Todos aquellos neurocirujanos jóvenes y no tan jóvenes que crean factible el que podamos modificar la actividad del sistema nervioso para conseguir efectos terapéuticos que mejoren la calidad de vida de nuestros pacientes e incluso podamos mejorar algún día las capacidades neurales de las personas sanas, deberían relacionarse rápidamente con este enorme campo de la neurociencia que es la NMQ.

Nuestra sociedad científica, la Sociedad Española de Neurocirugía, debería recoger toda la información existente sobre NMQ, alguna de la cual aquí se ha intentado plasmar, para que aquéllos que la dirigen actualmente, los que científicamente influyen en ella y los que "políticamente también influyen", sepan potenciar adecuadamente este nuevo campo que se abre para nuestra especialidad, pero sin olvidar que se trata realmente de un campo multidisciplinar en el que tendremos que saber movernos entre las diferentes ramas de las neurociencias, la bioingeniería, la industria y la bioética. Bienvenidos a la neurocirugía del tercer milenio.

\section{Bibliografía}

1. Abejon, D., Reig, E., del Pozo, C., Contreras, R., Insausti, J.: Dual spinal cord stimulation for complex pain: Preliminary study. Neuromodualtion 2005; 8: 105-111.

2. Alegret, M., Valdeoriola, F., Tolosa, E., Vendrell, P., Junque, C., Martinez, J., Rumiá, J.: Cognitive effects of unilateral posteroventral pallidotmy a 4-year follow-up study. Mov Disord 2003, 18; 323-328.

3. Alegret, M., Valdeoriola, F., Marti, M., Pillen, M., Junque, C., Rumia, J., Toosa, E.: Compartive cognitive effects of bilateral subthalamic stimulation and subcutaneous continuous infusión of apomorhine in Parkinson's disease. Mov Disord 2004, 19: 1463-1469.

4. Anderson Ahmed, A.: Treatment of patients with intractable obssesive-compulsive didorder with anerior capsular stimulation. Case report. J Neurosurg 2003. 98: 1104-1108.

5. Ansari, S., Chaudhri, K., Al Moutaery, K.: Vagus nerve stimulation: indications and limitations. Acta Neurochir Suppl 2007; 97: 281-286.

6. Aouizerate, B., Cuny, E., Martin-Guehl, D., Amieva, H., Benazzouz, A., et al.: Deep brain stimulation of the vetral caudate nucleus in the treatment of obssesive-compulsive disorder and major depression. Case report. J Neurosurg 2004; 101: 682-686.

7. Arechaga, C., Franco, M.L., Rodríguez, E.: Safety and efficacy of intrathecal baclofen infusión by implantable pump for the treatment of severe spinal spasticity: a Spanish Multicenter study. 2000. Neuromodulation Vol. 3 n ${ }^{\circ}$ 4; 175-182.

8. Bahra, A., Matharu, MS., Buchel, C., et al.: Brainstem activation specific to migraine headache. Lancet 2001; 35: 1016-1017.

9. Barcia-Salorio, JL., Roldán, P., Talamantes, F., PascualLeone, A.: Electrical inhibition of basal ganglio nucleus in Parkinson's disease: long-term results. Stereotac Funct Neurosurg 1999; 72: 202-207.

10. Barraquer-Bordas, L., Molet, J., Pascual-Sedano, B., Catala, H.: Delayed central pain associated with a sub-insular hematoma followed by parieto-occipital tumor. Favorable effects of chronic stimulation of the ventral posterior thalamic nucleus. Rev Neurol 1999; 29: 1044-1048.

11. Benabid, AL., Pollak, P., Loveau, A., Henry. S., de Rougemont, J.: Combined (thalamotomy and stimulation) stereotactic surgery of the VIM thalamic nucelus for biateral Parkinson's disease. Appl Neurophisiol 1987; 50: 344-346.

12. Benabid ,AL., Pollak, P., Gross, C., Hoffmann, D., Benazzouz, A., Gao, D.M., et al.: Acute and long-term effects of subthalamic nucleus stimulation in Parkinson's disease. Stereotac Funct Neurosurg 1994; 62: 76-84.

13. Benabid, A.L., Minotti, L., Koudsie, A., de San Martin, A., Hirsch, E.: Antiepileptic effect of high-frequency stimulation of the subthalamic nucleus (corpus Luysi) in a case of medically intractable epilepsy caused by focal dysplasia: a 30 months follow-up: Technical case report. Neurosurgery 2002; 50: $1385-1392$.

14. Beric, A., Nelly, P.J., Rezai, A., Sterio, D., Mogielner, A.., et al.: Complications of deep brain stimulation surgery. Stereotac Funct Neurosurg 2001; 77: 73-78.

15. Broseta, J., Rodin, P., González-Darder, J., Bordes, V., Barcia-Salorio, J.L.: Chronic epidural dorsal column stimulation in the treatment of causalgic pain. Appl Neurophisiol, 1982; 45: 190-194.

16. Broseta, J., Barbed, J., de Vera, J.A., Barcia-Salorio, J.L., García-March, G., González-Darder, J., Robaina, F., Joanes.: Spinal cord stimulation in peripheral arterial disease. A cooperative study. J. Neurosurg, 1986; 64: 71-80.

17. Broseta, J., García-March, G., Sánchez-Ledesma, M.J., Goncalves, J., Silva, I., Barcia, J.A., Llácer, J.L., BarciaSalorio, J.L.: High-cervical spinal cord electrical stimulation in brain low perfussion syndromes: Experimental basis and preliminary clinical report. Sterotac Funct Neurosurg 1994; 62: 171-178.

18. Burns, B., Watkins, L., Goadsby, P.: Treatment of medically intractable cluster headache by occipital nerve stimulation: long-term follow-up of eight patients. 2007; www.thelancet.com DOI:10.1016/S0140-6736(07)60328-6.

19. Butson, C.R., Noecker, A.M., Maks, C.B., McIntyre, CC.: Stim Explorer deep brain stimulation parameter selection software system. Acta Neurochir Suppl 2007; 97: 569-574. 
20. Canavero, S., Bonicalzi, V.: Extradural cortical stimulation for central pain. Acta Neurochir Suppl 2007; 97: 27-36.

21. Cervera-Paz, F.J., Manrique, M.: Auditory brainstem implants: past, present and future prospects. Acta Neurochir Suppl 2007; 97: 437-442.

22. Cioni, B.: Motor cortex stimulation for Parkinson's disease. Acta Neurochir Suppl 2007; 97: 233-238.

23. Clavo, B., Robaina, F., Catala, L. et al.: Effect of cervical spinal cord stimulation on regional blood flow and oxygenation in advanced head and neck tumors. Annals of Oncology 2004; 15: 802-807.

24. Coffey, R.J., Lozano, A.M.: Neurostimulation for chronic noncancer pain: an evaluation of the clinical evidence and recommendations for future trial designs. J Neurosurg 2006; 105: 175-189.

25. Cooper, I.S., Amin, I., Rielan, M., Waltz, J.M., Poo, T.P.: Chronic cerebellar stimulation in epilepsy. Clinical and anatomical studies. Arch Neurol 1976; 33: 559-570.

26. Coubes, P., Cif, L., El Fertit, H., Hemm, S. et al.: Stimulation of the globus pallidus internus in patients with primary generalized dystonia : long-term results J Neurosurg 2004; 101: 189-194.

27. De Ridder, D., De Mulder, G., Verstraeten, E. et al.: Auditory cortex stimulation for tinnitus. Acta Neurochir Suppl 2007; 97: 451-462.

28. De Ridder, D., De Mulder, G., Walsh, V., et al.: Magnetic and electrical stimulation of the auditory cortex for intractable tinnitus. Case report. J Neurosurg 2004; 100: 560-564.

29. Di Girolamo, S., Napolitano, B., Alessandrini, M., Bruno, E.: Experimental and clinical aspects of the efferent auditory system. Acta Neurochir Suppl 2007; 97: 419-424.

30. Escamilla-Sevilla, F., Minguez-Castellanos, A., Katati, M.J., Martin-Linares, J.M., Meersmans, M., Ortega-Moreno, A., Mona, V.: Clinical utility of deep brain stimulation in an advanced Parkinson's disease. Neurología 2004; 19: 19-27.

31. Fernández-González, F., Seijo, F., Menéndez-Guisasola, L, Salvador, C., Roge,r RL., González-García, F.J. y cols.: Stereotactic target identification for neurosurgery of Parkinson disease. Rev Neurol 1999; 27: 600-608.

32. Figueiras-Mendez, R., Regidor, I., Riva-Meana, G., Magariños-Ascone, C.M.: Further supporting evidence of benefical subthalamic stimulation in Parkinson's disease. Neurology 2002; 58: 469-470.

33. Figueiras-Mendez, R., Marin-Zarza, F., Molina, A., Jiménez-Jimenez, F.J., Ortí-Pareja, M., Magariños, C., LopezPino, M., Martinez, V.: Subthalamic nucleus stimulation improves directly levodopa induced dyskinesias in Parkinson's disease. J Neurol Neurosurg Psychiat 1999; 66: 549-550.

34. Fodstad, H., Haritz, M.: Electricity in the treatment of nervous system disease. Acta Neurochir Suppl 2007; 97: 1119.

35. Fountas, K.N., Smith, J.R.: A novel closed-loop stimulation system in the control of focal medically refractory epilepsy. Acta Neurochir Suppl 2007; 97: 357-362.

36. Franzini, A., Ferroli, P., Leone, M., Broggi, G.: Stimulation of the posterior hypothalamus for treatment of chronic intratable cluster headaches. The first reported series. Neurosurgery 2003. 52: 1095-1101.

37. Franzini, A., Marras, C., Ferroli, P., Bugiani, O., Broggi, G.: Stimulation of the posterior hipothalamus for medically intractable impulsive and violent behaviour. Stereotact Funct Neurosurg 2005; 83: 63-66.

38. Franzini, A., Marras, C., Tringali, G., Leone, M et al.: Chronic hihg frequency stimulation of the posteromedial hypothalamus in facial pain sybdromes and behaviour disorders. Acta Neurochir Suppl 2007; 97: 399-406.

39. Garcia-Larrea, L., Maarrawi, J., Peyron, R., Costes, N., Mertens, P., Magnin, M., Laurent, B.: On the relation between sensory deafferentation,pain ant thalamic activity in Wallenberg's syndrome: A PET-scan study before and after motor cortex stimulation. European Journal of Pain, 2006; 10: 677-688.

40. Gildenberg, P.L.: Neurosurgical statement on neuroaugmentive devices. Appl Neurophysiol 1977; 40: 69-71.

41. Gironell, A., Amirian, G., Kulisysky, J., Motet, J.: Usefulness of an introperative electrophysiological navigator system for subthalamic nucelus surgery in Parkinso's disease. Stereotac Funct Neurosurg 2005; 83: 101-107.

42. González-Darder, J., Canela, P., González-Martínez, V.: High cervical spinal cord stimulation for unstable angina pectoris. Stereotac Funct Neurosurg, 1991; 56: 20-27.

43. Green, L.A., Wang, S., Owen, S.L., Aziz, T.: The periacueductal grey area and the cardiovascular system. Acta Neurochir Suppl 2007; 97: 521-528.

44. Guridi, J., Lozano, AM.: A brief history of pallidotomy. Neurosurgery 1997; 41: 1169-1180.

45. Guridi, J., Obeso, JA.: The role of subthalamic nucleus in the origin of hemiballism and parkinsonism: new surgical perspective. Adv Neurol 1997; 74; 235-247.

46. Guridi, J., Rodriguez-Orz, MC., Ramos, E., Linazasoro, G., Obeso, J.A.: Discrepancy between imaging and neurophisiology in deep brain stimulation of medial pallidum and subthalamic nucelus in Parkinson's disease. Neurología 2002; 17: 183-192.

47. Hariz, M.I., Fodstad, H.: Do microelectrode techniques increase accuracy or decrease risks in pallidotomy and deep brain stimulation? A critical review of the literature. Stereotact Funct Neurosurg 1999; 72: 157-169.

48, Hariz, M,I., Shamsgovara, P., Johansson, F., Harz, G.M., Fodstad, H.: Tolerance and tremor rebound following long term chronic thalamic stimulation for parkinsonian and essencial tremor. Stereotact Funct Neurosurg 1999; 72: 208218.

49. Holloway, K., Gaede, S.E., Starr, P., Rosenow, J., Ramakrishnan, V., Henderson, J.: Frameless stereotaxy using bone fiducial markers for deep brain stimulation. J Neurosurg 
2005; 103: 404-413.

50. Hosobuchi ,Y., Adams, J.E., Rutkins, B.: Chronic thalamic stimulation for the control of facial anestesia dolorosa. Arch Neurol 1973. 29: 158-161.

51. Jiménez, F., Velazco, F., Salin-Pascual, R., Hernandez, J.A., et al.: A patient with resistant major depresión disorder treated with deep brain stimulation in the inferior thalamic peduncle. Neurosurgery 2005; 77: 159-162.

52. Jiménez, F., Velasco, F., Salín-Pascual, R., Velasco, M., et al.: Neuromodulation of the inferior thalamic peduncle for major depresión and obsseive compulsive disorder. Acta Neurochir Suppl 2007; 97: 393-398.

53. Johnson, M.D., Burchiel, K.K.: Peripheral stimulation for treatment of trigeminal postherpetic neuralgia and trigeminal posttraumatic neuropathic Pain: a pilot study. Neurosurgery 2004; 55: 135-141.

54. Katayama, Y., Yamamoto, T., Kobayashi, K., Kasay, M., et al.: Motor cortex stimulation for phantom limb pain: Comprehensive therapy with spinal cord and thalamic stimulation. Stereotac Funct Neurosurg 2001; 77: 159-162.

55. Karceski, S.: Electrical stimulation devices in the treatment of epilepsy. Acta Neurochir Suppl 2007; 97: 247-259.

56. Kennedy, P., Bakau, R., Moore, M., Adams, K., Goldwaith, J. Direct control of a computer from the human central nervous system. IEEE Trans Rehab Eng 2000; 8: 198-202.

57. Kennedy, P., Andreasen, D., Ehirim, P., King, B. et al.: Using human extra-cortical local field potentials to control a switch. J Neur Eng 2004; 1: 72-77.

58. Khan, Y.N., Raza, S.S., Khan, E.A.: Application of spinal cord stimulation for the treatment of abdominal visceral pain syndcromes: Case reports. Neuromodulation 2005; 8: $14-$ 27.

59. Krames, E.S.: The Right place at the right time. Neuromodulation 2005: 8: 149-152

60. Krames ,E.S.: The "New Look and Feel" of Neuromodulation. Neuromodulation: Technology at the neural interface 2007; 10: 1-3.

61. Kumar, K., Jefferson, R., Wilson, R., Taylor, R.S., Gupta, S.: Complications of spinal cord stimulation, suggestions to improeve outcome, and financial impact. J Neurosurg Spine 2006; 5: 191-203.

62 . Kutzenberger, J.: Surgical therapy of detrusor overactivity (hyperreflexia) in paraplegic patients by sacral deafferentation and implant driven micturition by sacral anterior root stimulation: medthods, indications, results, complications, an future prospects. Acta Neurochir Suppl 2007; 97: 333-339.

63. Leone, M., Franzini, A., Broggi, G., Bussone, G.: Hypothalamic stimulation for intractable cluster headache: Long term experience. Neurology 2006; 67: 150-152.

64. Li, J., Andrews, RJ.: Trimodal nanoelectrode array for precise deep brain stimulation: prospects of a new technology based on carbon nanofiber arrays. Acta Neurochir Suppl 2007. 97: 537-545.
65. Lozano, A.M.: Deep-brain stimulation: Challenges to integrating stimulation technology with human neurobiology, neuroplasticity and neural repair. J Rehabil Res Dev 2001; 38: 10-19.

66. Magis, D., Allena, M., Bolla, M., De Pasqua, V., Remacle, J.M., Schoenen, J.: Occipital nerve stimulation for drug-resistant chronic cluster headache: a prospective pilot study. 2007; http://neurology.thelancet.com. DOI: 1016/S 1474-442270058-3.

67. Manola, L., Holsheimer, J.: Motor cortex stimulation: role of computer modeling. Acta Neurochir Suppl 2007; 97: 497-503.

68. Matharu, M.S., Bartsch, T., Ward, N., Frackoviak, R.S., Weiner, R., Goadsby, P.J.: Central neuromodulation in chronic migraine patients with suboocipital stimulators: a PET study. Brain 2004; 127: 220-230.

69. Mayberg, H.S., Lozano, A.M., Voon, V., McNeely, H., et al.: Deep brain stimulation for treatment-resistant depression. Neuron 2005. 45: 651-660.

70. Meloy, S.: Neurally augmented sexual function. Acta Neurochir Suppl 2007; 97: 359-363.

71. Minguez-Castellanos, A., Escamilla-Sevilla, F., Katati, MJ., Martin-Linares, JM., Meersmans, M., Ortega-Moreno, A., Arjona, V.: Diffrent paterns of medication change after subthalamic or pallidal stimulation for Parkinson's disease: a target related effect or selection bias? J Neurol Neurosurg Psichiat 2005; 76: 34-39

72. Miocinovic, S., Noecker, A.M., Maks, C.B., Butson, C.R., McIntyre, C.C., Cicerone: Stereotactic neurophysiological recording and deep brain stimulation electrode placement software system. Acta Neurochir Suppl 2007; 97: 561-567.

73. Mogilner, A.Y., Rezai, A.R.: Brain stimulation: History, current clinical application, and future prospects. Acta Neurochir Suppl 2003; 87: 113-120.

74. Morita, I., Keith, M.W., Kanno, T.: Dorsal column stimulation for pesistent vegetative state. Acta Neurochir Suppl 2007; 97: 455-459.

75. Muller-Putz, G.R., Scherer, R., Pfurtscheller, G., Rupp, R.: EEG-based neuroprosthesis control: a step towards clinical practice. Neurosci Left 2005; 382: 169-174.

76. Nguyen, J.P., Lefaucheur, J.P., Keravel, Y.: Motor cortex stimulation. Electrical Stimulation and the Relief of Pain. Pain Research and Clinical Management.; Vol. 15 B.A. Simpson Ed. Elsevier Science 2003; 197-209.

77. Nikita, K.S., Tsirogiannis, G.L.: Computacional models simulating electrophysiological activity in the basal ganglia. Acta Neurochir Suppl 2007; 97: 505-511.

78. North, R.: Definition of neuromodulation. www. neuromodulation.com 2006.

79. Oh, M.Y., Abosch, A., Kim, S.H., Lang, A,E., Lozano, A.M.: Long-term hardware-related complications of deep brain stimulation. Neurosurgery 2002; 50: 1268-1276.

80. Operative Neuromodulation Volume 1: Functional 
Neuroprostetics Surgery. Surgery. Sakas D.E., Simpson B.A., Krames E.S (eds.). 2007. Springer-Verlag/Wien.

81. Operative Neuromodulation Volume 2: Neural Networks Surgery. Sakas D.E., Simpson B.A., Krames E.S (eds.). 2007. Springer-Verlag/Wien.

82. Ortega-Moreno, A., Arjona, V.: Different patterns of medication change after subthalamic or pallidal stimulation for Parkinson's disease: target related effect or selection bias? J Neurol Neurosurg Psychiat 2005; 76; 34-39.

83. Park, M.C., Goldman, M.A., Carpente,r L.L, et al.: Vagus nerve stimulation for depresión: rationale, anatomical and physiological basis of efficacy and future prospects. Acta Neurochir Suppl 2007; 97: 407-416.

84. Pfurtscheller, G., Muller, G.R., Pfurtscheller, J., Gerner, H.J., Rupp, R.: "Though"-control of functional eletrical stimulation to restore hand grasp in a patient with tetraplegia. Neurosci Lett 2003; 351: 33-36.

85. Rainov, N., Heidecke, V.: Motor cortex stimulation for neuropathic facial pain. Neurological Research; 2003; Volume 25: 157-161.

86. Rasche, D., Ruppolt, M., Stippich, C., Unterberg, A., Tronnier, V.M.: Motor cortex stimulation for long-term relief of chronic neuropathic pain: A 10 year experience. Pain 2006; 121: 43-52.

87. Ratto, C., Parello, A., Donisi, L., Doglietto, G.B.: Sacral neuromodulation in the treatment of defecation disorders. Acta Neurochir Suppl 2007; 97: 341-350.

88. Robaina, F.J., Rodriguez, J.L., de Vera, J.A., Martin, M.A.: Transcutaneous electrical nerve stimulation and spinal cord stimulation for pain relief in reflex sympathetic dystrophy. Stereotac Funct Neurosurg 1989; 52: 53-62.

89. Robaina, F., Dominguez, M., de Vera, J.A. et al.: Spinal cord stimulation for pain relief in vasospastic disorders of the upper limbs. Neurosurgery, 1989; 24: 63-68.

90. Robaina, F., Calvo, B., Catalá, L., et al.: Blood Flow Increase by Cervical Spinal Cord Stimulation in Middle Cerebral and Common carotid Arteries. Neuromodulation 2004; 7 : 26-31.

91. Robaina, F., Clavo, B.: Spinal cord stimulation in the treatment of post-stroke patients: current state and future directions. Acta Neurochir Suppl 2007; 97: 277-282.

92. Robaina, F., Clavo, B.: The role of spinal cord stimulation in the management of patients with brain tumors. Acta Neurochir Suppl 2007; 97: 445-453.

93. Rodríguez, M., Aldaya, C., de la Torre, R.: Indicaciones y evidencias clínicas en la aplicación terapéutica de la infusión espinal. Rev. Soc. Esp. Dolor 2003; 10: Supl. I: 19-32.

94. Rosal, S.K.: Predictable social and ethical consequences of cognitive technical systems in health care. Symposium on Technical Cognitive Systems in health and medicine. 2005. September $12^{\text {th }}-13^{\text {th }}$, Nuremberg, Germany.

95. Rosenow, J.M., Stanton-Hicks, M., Rezai ,AL., Henderson, J.M.; Failure modes of spinal cord stimulation hard- ware. J Neurosurg Spine 2006. 5: 183-190.

96. Roth, R.M., Flashman, L.A., Saykin, A.J., Roberts, D.W.: Deep brain stimulation in neuropsychiatric disorders. Current Psychiatry Reports 2001; 3: 366-372.

97. Rupp, R., Gerner, H.J.: Neuroprosthetics of the upper extremity-clinical application in spinal cord injury and challenges for the future. Acta Neurochir Suppl 2007; 97: 419-426.

98. Sabih, P., Strafella, A., Dostrovsky, J.O., Lozano, A., Lang, A.E.: Immediate motor effects of stimulation through electrodes implanted in the human globus pallidus. Stereotact Funct Neurosurg 1998; 70: 1-18.

99. Sakas, D.E., Panourias, I.G., Simpson, B.A., Krames, E.S.: Preface. Acta Neurochir Supp 2007; 97.

100. Sakas, D.E., Panourias, I.G., Simpson, B.A., Krames, E.S.: An introduction to operative neuromodulation and functional neuroprosthetics, the new frontiers of clinical neuroscience and biotechnology. Acta Neurochir Supp 2007; 97: 3-10.

101. Sakas, D.E., Panourias, I.G., Singounas, E., Simpson, B.A.: Neurosurgery for pschiatric disorders: from the excision of brain tissue to the chronic electrical stimulation of neural networks. Acta Neurochir Suppl 2007; 97: 365-374.

102. Salvador-Aguiar, C., Menendez-Guisasola, L., Blázquez-Estrada, M., Fernandez-Gonzalez, F., Seijo-Fernandez, F.: Psychiatric symptoms of Parkinson's disease following deep brain stimulation surgery on the subthalamic nucleus. Rev Neurol 2004; 39; 651-655.

103. Shoenen, J., Di Clemente, L., Vandenheede, M., et al. : Hypothalamic stimulation in chronic cluster headache: apilot study of efficacy and mode of action. Brain 2005; 128: 940947.

104. Sikström, S.: Computacional perspectives on neuromodulation of aging. Acta Neurochir Suppl 2007; 97: 513518.

105. Slavin, K.V., Wess, C.: Trigeminal Branch stimulation for intractable neuropathic pain: Technical note. Neuromodulation 2005; 8: 7-13.

106. Slavin, K.V.: Periheral nerve stimulation for the treatment of neuropathic craneofacial pain. Acta Neurochir Supp 2007; 97: 115-120.

107. Starr, P.A., Barbaro, N.M., Raskin, N.H., Ostrem, J.L.: Chronic stimulation of the posterior hypothalamic region for cluster headache: technique and 1-year results in foru patients. J Neurosurg 2007; 106: 999-1005.

108. Stieglitz, T.: Neural prostheses in clinical practice: biomedical mycrosistems in neurological rehabilitation. Acta Neurochir Suppl 2007; 97: 411-418.

109. Stieglitz, T.: Restoration of neurological functions by neuroprosthetic technologies: future prospects and trends towards micro-nano-, and biohybrid systems. Acta Neurochir Suppl 2007; 97: 435-442.

110. Tsubokawa, T., Yamamoto, T., Katayama, Y., Hirayama, T., et al.: Deep-brainstimulation in a persistent vege- 
tative state: follow-up results and criteria for selection of candidates. Brain Inj 1990; 4: 315-327.

111. Tsubokawa, T., Katayma ,Y., Yamamoto, T., Hyrayama, T., Koyama, S.: Chronic motor cortex stimulation for the treatment of central pain. Acta Neurochir Suppl 1991; 52: 137-139.

112. Turner, J.A., Loeser, J.D., Deyo, R.A., Sanders, S.B.: Spinal cord stimulation for patients with failed back surgery syndrome or complex regional pain syndrome: a systematic review of effectiveness and complications. Pain 2004; 108: 137-147.

113. Upadhyaya, C.D., Sagher, O.: Cervical spinal cord stimulation in cerebral ischemia. Acta Neurochir Suppl 2007; 97: 267-275.

114. van Kuyck, K., Gabriéls ,L., Cosyns, P., Arckens, L., Sturm, V., Rasmussen, S., Nuttin, B.: Behavioural and physiological effects of electrical stimulation in the nucleus accumbens: a review. Acta Neurochir Suppl 2007; 97: 375-391.

115. Velasco, F., Velazco, M., Velazco, A.L., Menez, D., Rocha, L.: Electrical stimulation for epilepsy: stimulation of hippocampal foci. Stereotac Funct Neurosurg 2001; 77: 223227

116. Vidal, J., Fenollosa, P., Martin, E., Barbed, J., Robaina, F., Fuentes, M., Rodriguez-Palmero, M., CerdáOlmedo, G., De Andrés, J.: Safety and efficacy of intrathecal baclofen infusion by implantable pump for the treatment of spinal spasticity: a Spanish Multicenter Study. Neuromodula- tion 2000; 3: 175-182.

117. Vignes, J.R., Bauchet, L., Ohanna, F.: Dorsal rhizotomy combined with anterior sacral root stimulation for neurogenic bladder. Acta Neurochir Suppl 2007; 97: 323-331.

118. Vignes, J.R., de Seze, M., Guerin, J., Barat, M.: Sacral neuromodulation as a functional treatment of bladder overactivity. Acta Neurochir Suppl 2007; 97: 315-322.

119. Viola, M.V., Patrinos, A.A.: A neuroprosthesis for restoring sight. Acta Neurochir Suppl 2007; 97: 481-486.

120.Visser- Vandewalle, V., Temel, Y., Boon, P., Vreeling, F., et al.: Chronic bylateral thalamic stimulation, a new therapeutical approach in intractable Tourette syndrome. Report of three cases. J Neurosurg 2003; 99: 1094-1100.

121. Warwick, K. I Ciborg.: University of Illinois Press. 2004.

122. Weiner, R.L.: Occipital neurostimulation for treatment of intractable headache syndromes. Acta Neurochir Suppl 2007; 97: 129-133.

Robaina-Padrón, F.J.: Neuromodulación quirúrgica. Nuevos horizontes en Neurocirugía. Neurocirugía 2008; 19: $143-155$.

Correspondencia postal: Francisco Javier Robaina Padrón. Unidad del Dolor Crónico y Neurocirugía Funcional. Hospital Universitario de Gran Canaria Doctor Negrín. C) Barranco de la Ballena S/N, 35010 Las Palmas de Gran Canaria. 